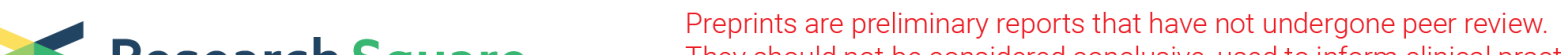 Research Square They should not be considered conclusive, used to inform clinical practice, or referenced by the media as validated information.
}

\section{Matrine-chitosan hydrogels for treating subclinical bovine mastitis by intramammary infusion-effect on milk microbiome and metabolites}

\section{Fuxin Wu}

Beijing university of Agriculture

Hua Zhang

Beijing University of Agriculture

Hui Niu

Beijing university of Agriculture

\section{Benhai Xiong}

University of the Chinese Academy of Sciences

Jinjin Tong

beijing university of Agriculture

Linshu Jiang ( $\sim$ linshujiangbua@163.com )

Beijing University of Agriculture

\section{Research article}

Keywords: matrine-chitosan hydrogels, inflammatory factors, microbiome, metabolomics, subclinicalmastitis, dairy cows

Posted Date: May 21st, 2020

DOl: https://doi.org/10.21203/rs.3.rs-26655/v1

License: (c) (1) This work is licensed under a Creative Commons Attribution 4.0 International License. Read Full License 


\section{Abstract}

\section{Background}

The metabolic processes of cows undergo significant changes during subclinical mastitis, but their molecular mechanisms have not been clearly elucidated. This study investigated the changes in milk metabolites after intramammary infusion of matrine, a plant alkaloid with anticancer properties, in the form of a chitosan hydrogel into bovine mammary glands with subclinical mastitis. Infusions were continued for seven days, and milk samples were collected on day 1 and day 7 for analysis of the microbiome by $16 \mathrm{~S}$ rRNA gene sequencing and metabolites by liquid chromatography-mass spectrometry.

Results

Matrine-chitosan hydrogels (MCHs) significantly decreased the somatic cell count on day 7 and the Simpson index indicated that microbial diversity was significantly lower on day 7 than on day 1 . On day 7, the numbers of Aerococcus, Corynebacterium_ 1 and Staphylococcus were significantly lower, while the abundance of Firmicutes was very significantly decreased. The numbers of Probacteria increased, however. In milk samples, we identified 74 differentially expressed metabolites and the MCH infusion group had the most significantly upregulated metabolites including sphingolipids, glycerophospholipids, flavonoids and fatty acyls. Principal component analysis and the orthogonal partial least squares discriminant test confirmed good separation of the milk metabolites. The identification of active milk metabolic pathways after $\mathrm{MCH}$ treatment supported the known antimicrobial and anti-inflammatory properties of matrine that are associated with glycerophospholipid metabolism and the sphingolipid metabolic signaling pathways.

\section{Conciusions}

These insights into the mechanisms and the corresponding biological responses to matrine demonstrate its potential immunoregulatory activity and emphasize the need for continued investigation.

\section{Background}

The udder microbiota plays an important role in the host-pathogen interactions of the innate and adaptive immune system. This is especially true of pathogens that trigger inflammation that is detrimental to mammary tissue and bovine physiology [1]. Mastitis is an inflammation of the udder most commonly from bacterial infection, and the pathological changes not only affect milk quality but also quantity [2]. The increasing demand for animal protein has led to substantial growth in the use of antibiotics in food animal production [3], which can cause antimicrobial resistance that is a global concern for treating human as well as farm animal diseases. Developing an effective drug for mastitis that is safe for use in food animals and for which no resistance exists in the microbial population would be highly desirable for the worldwide dairy industry. 
Matrine is a polycyclic alkaloid isolated from the plant, Sophora flavescens that has been used in Chinese traditional medicine because of its anticancer, anti-inflammatory, cardioprotective, and opioid effects [4]. Previous research [5] found that matrine inhibited the virulence of Staphylococcus aureus, one of the main pathogens of mastitis. Furthermore, matrine attenuated the lipopolysaccharide-induced immune response by downregulating IL-1 and IL-17 and inhibiting production of the proinflammatory compound, malondialdehyde, reducing inflammation and oxidative stress, and enhancing CCR7 expression [6, 7]. Chitosan is a natural polymer produced by acetylation and enzymatic cleavage of chitin, which is the most abundant animal polysaccharide in nature.[8]. The applications for chitosan have continued to increase because of the abundance of its source, its biocompatibility and ease of chemical modification. Studies have also shown that chitosan has anti-inflammatory, antimicrobial, cholesterol-lowering, immunomodulatory, and antitumor properties [5]. Chitosan can be beneficial at the cellular or molecular level [9], by reducing intracellular material leakage and triggering an antimicrobial response to enhance the therapeutic effects of mastitis treatment. Chitosan can be formulated to be injectable at room temperature but change into a biodegradable hydrogel at body temperature [10]. It has been used in drug formulations and delivery vehicles for decades; however, the influence of matrine and chitosan on the udders of dairy cows has received little research attention. Therefore, it is worthwhile to explore the effectiveness of intramammary infusion of matrine-loaded chitosan hydrogels ( $\mathrm{MCHs}$ ) on the udders of dairy cows with mastitis in terms of positively changing the microbial population and metabolic profile of milk. We hypothesized that intramammary injection of $\mathrm{MCHs}$ would favor the growth of beneficial microorganisms and cause a shift in the milk microbiota in normal udder quarters.

DNA sequencing has become routine for many labs and the advanced software and bioinformatics databases available have created great opportunities for studying pathogenic mechanisms [11, 12]. By understanding the host responses to microbial attack, we hope to be able to identify the most effective targets for antimicrobial intervention, to develop new treatments for bovine mastitis and to find sensitive biomarkers for early detection and diagnosis. Metabolomics can also be used for quantitative measurement of the metabolic state of milk, including the biomarkers of lactation [13], the variation in metabolites associated with mastitis[14], and differences in metabolism after antibacterial therapy [15]. Little is known about the changes in milk metabolites in response to $\mathrm{MCH}$ treatment of mastitis, and one of the goals of this study was to obtain detailed information on the effect of matrine delivery by chitosan hydrogel injection into bovine mammary glands on milk microbiota and metabolites. We investigated the effects $\mathrm{MCH}$ on subclinical-mastitis and the relationship between milk microbiota diversity and metabolite profiles, to probe the mechanisms of the antibacterial benefits of matrine and chitosan for mastitis, and to provide new knowledge for the development of novel, safe and effective prophylactic and therapeutic compounds for dairy cow operations.

\section{Results}

\subsection{Somatic cell counts in milk}


Matrine-chitosan hydrogels significantly decreased the somatic cell count on day 7 compared to day 1 ( $p$ <0. 05) (Fig. 1).

\subsection{Diversity and relative numbers of milk microbiota}

A total of 1,117,565 high-quality sequences from twelve samples passed quality control and could be used for testing. The sequences averaged $308 \mathrm{bp}$, and there was greater than ninety-nine percent depth coverage; therefore, the amount of data was sufficient to show differences in all bacterial species. The adiversity indices of the microbiota are shown in table 1. Although ACE showed a tendency to decrease, no different was observed in the Chao indices, which were all representative of bacterial community richness. Moreover, the Simpson index showed that the bacterial diversity at day 7 was significantly lower than at day 1 ( $p \otimes 0.05)$, and there was an overall tendency to decrease $(p=0.06)$, but this is variable and depends on if the calculations are based on abundance or biomass. No significant differences were seen among the groups for the other a-diversities.

Principal component analysis (PCA) on the microbial populations present on day 1 vs day 7 showed clear differences, and the weighted UniFrac values were also determined (Fig. 2). Principal coordinates one and two were found to account for 51.76 and $26.2 \%$ of total variance, confirming that the milk microbiome after seven days of $\mathrm{MCH}$ infusion was remarkably distinct from that on day one.

The taxonomic changes in milk samples from day 1 to day 7 were determined. Proteobacteria, Firmicutes, Actinobacteria and Bacteroidetes were the three predominant phyla (Fig. 4A). The abundance of Firmicutes was extremely significantly decreased on day $7(p=0.01)$, whereas the numbers of Proteobacteria increased ( $p=0.01$ ) (Fig. 3A, B). Taxa with a relative abundance of one percent in at least one sample were identified, and the ten most abundant genera are presented (Fig. 3C, D). On day 7, there was a significantly lower abundance of Aerococcus $(p=0.01)$, Corynebacterium_1 $(p=0.08)$ and Staphylococcus $(p=0.03)$. In contrast, the relative abundances of Pseudomonas $(p=0.01)$ and Ralstonia $(p=0.02)$ were significantly increased.

\subsection{Identification and comparison of milk metabolites}

A non-targeted metabolome method was used to evaluate milk metabolites after MCH treatment. Reproducible metabolite data profiles (1001) were obtained and differences between day 1 and day 7 were characterized using VIP metrics from OPLS-DA. Seventy-four milk metabolite signals were obtained that were significantly different between $\mathrm{MCH}$-treated and untreated cows (VIP > 1 and $p<0.05)($ table 2$)$. They consisted mainly of sphingolipids, oxanes, glycerophospholipids, flavonoids and fatty acyls. Milk from animals receiving MCHs for seven days had higher levels of steroids and steroid derivatives, prenol lipids, oxanes, macrolides and analogues, hydroxy acids and derivatives, carboxylic acids and derivatives and Benzene and substituted derivatives than milk on day 1.

PCA and OPLS-DA were employed to evaluate the differences in metabolomes. The PCA scores showed clear differences between day 1 and day 7 milk samples (Fig. 4A). Principal components one and two were found to account for $31.70 \%$ and $16.10 \%$ of the variation. The variables for assessing OPLS-DA 
model quality are shown in validation plots (Fig. 4B). The Q2 value of the OPLS-DA model was 0.939 , and the $\mathrm{R} 2 \mathrm{Y}$ value was 0.963 . Q2 represents the model's prediction ability and the closer these 3 indicators are to 1, the more stable and reliable it is. Q2 > 0.5 indicates that the model's prediction ability is good, while Q2 $<0.5$ indicates poor predictability. The OPLS-DA data confirmed that the two groups had significantly different types and levels of metabolites (Fig. 4C). OPLS-DA model integrity is shown by validation plots. The day 7 metabolite profiles were significantly different from those of day 1 indicating that the PCA and OPLS-DA results were valid for assessing variations in milk metabolomes between the two days.

\subsection{Differences in metabolites resulting from changes in metabolic pathways}

Comparison of metabolism via changes in pathway activation from $\mathrm{MCH}$ treatment on day 1 to 7 was performed using the Kyoto Encyclopedia of Genes and Genomes (KEGG) for determining enrichment of pathways involved in metabolite production (table 3; Fig. 5). Enrichment analysis showed that sphingolipid metabolism, the sphingolipid signaling pathway, glycerophospholipid metabolism and ABC transporters, were significantly affected by $\mathrm{MCH}$ injection.

Pathway topology analysis (Fig. 6) $(p<0.05)$ revealed 8 key pathways enriched between days 1 and 7 . They included sphingolipid, phenylalanine, glycerophospholipid, and starch and sucrose metabolism, synthesis of phenylalanine, tyrosine and tryptophan and linoleic acid metabolism.

\section{Discussion}

The results of this study revealed that MCHs markedly decreased somatic cell count after 7 days of treatment. Although we did not observe any changes in bacterial community richness by the Chao indices, the Simpson index showed a significant decrease in the bacterial diversity. In particular, on day 7 , the relative abundance of Firmicutes was significantly decreased, and that of Proteobacteria was significantly increased compared to levels on day 1 . It has been reported that the richness and diversity of the milk microbiome reflects the health of the cows and functional performance of the organs [16-18]. Antibacterials are commonly used to treat bovine mastitis, but their efficacy is unclear [19]. That is why we chose to test the plant-derived compound, matrine, as an intramammary treatment loaded into chitosan hydrogels to see if it would alter the milk microbiota and improve mammary gland function.

Measurement of the number of somatic cells in milk is an internationally recognized test for the detection of mastitis. A SCC of more than 200,000 per $\mathrm{ml}$ is considered to be evidence of disease[20]. Recent investigations have shown that the milk microbiota of dairy cows are usually closely associated with the SCC $[21,22]$. This suggests that modulation of the udder ecosystem through the microbiota could help to maintain homeostasis and enhance the mammary gland's defense mechanism [23,24]Consistent with these reports, we observed that the udder SCC was significantly increased by in mastitis by common pathogens like Staphylococcus, but was markedly decreased after perfusion of the udder with matrinechitosan hydrogel. This is in line with previous studies showing that chitosan hydrogels decreased the SCC in milk from dairy cows[25]. The therapeutic synergy achieved by loading the chitosan hydrogel with 
matrine suggests potential application in mastitis protection and treatment. The mechanism by which the milk microbiota influences inflammatory and immune responses to decrease the SCC still needs further investigation.

The next generation of high-throughput DNA sequencing methods together with updated bioinformatics software is now being used for the in-depth assessment of microbial communities to elucidate how bacterial activity and metabolites affect human and animal health [26]. We found that the microbial diversity in milk was dramatically decreased after one week of intramammary infusions of matrinechitosan hydrogels. The relative abundance of Proteobacteria was significantly increased, while Staphylococcus numbers decreased significantly. Staphylococci are internationally recognized as one of the most prevalent mastitis pathogens, accounting for about $70 \%$ of the cases [2].. Moreover, it has been reported that Proteobacteria are significantly more abundant in healthy cows than those with mastitis[2]. Previous studies also showed that the numbers of Proteobacteria after non-antibiotic hydrogel treatment increased significantly [27], indicating that hydrogel therapy can effectively return the milk microbial environment to normal diversity and defend against disease. Our investigation provides mechanistic insights into how the microbiota respond to $\mathrm{MCH}$ may help in the development of novel prophylactic and therapeutic products as alternatives to antibiotics in dairy cows. Our data are also important for understanding how the regulation of biosynthesis by milk microbiota influences udder health and defense against mastitis. The milk microbiota are usually closely associated with the SCC [21, 22]. suggesting that the udder microbiota plays an important part in modulation of the ecosystem and maintenance of homeostasis to resist mammary gland infections $[23,24]$

Metabolomics is a comparatively new research method that has been widely used in the detection of mastitis in recent years due to its more comprehensive test results. Previous studies suggested that metabolomics could provide a more complete understanding of an animal's physiology and biochemistry .[28]. The metabolomics data from this study highlight the potential function of matrine-chitosan hydrogels in modulating the metabolite levels, which were found to be enriched in sphingolipid metabolic pathways, phenylalanine, glycerophospholipid, and starch and sucrose metabolism. A previous report suggested that sphingolipid metabolites acted as signaling molecules to regulate a diverse group of cellular processes, particularly those related to immunity, inflammation and inflammatory disorders .[29]. We speculate that this activity might be associated with the decrease in SCC caused by matrine-chitosan hydrogel injection. The metabolic differences we observed gave us a further insight into how $\mathrm{MCH}$ affects metabolite levels before and after mastitis treatment, and showed that alterations in the milk metabolome could be used to reveal the therapeutic mechanism of matrine for mastitis treatment and in the recovery of milk production in dairy cows.

We found significant changes in concentration of a number of sphingolipids, glycerolipids, fatty acyls, glycerophospholipids, and organo-oxygen compounds that significantly differed from day 1 to day 7 . Sphingolipids are major components of cell membranes and are considered to be widely involved in important processes such as cell aging and apoptosis .[30]. Glycerolipids [30] and fatty acyls [32] are both important components of cell membranes. Previous research found that bacterial invasion can 
induce inflammatory reactions and cause oxidative damage during the development of the breast [33], accompanied by apoptosis .[34, 35]. The reduction in sphingolipids, glycerolipids, and fatty acyls seen in this study indicates that apoptosis caused by inflammatory reactions may have been weakened. The decrease of organooxygen compounds suggests that the oxidative damage caused by inflammatory reactions may also have been reduced. The metabolites were enriched in sphingolipid and glycerophospholipid metabolism[36], which are both related to apoptosis pathways.[37, 38]. Therefore, we conclude that $\mathrm{MCH}$ s can inhibit apoptosis by blocking oxidative damage, thereby reducing inflammatory changes to the udder.

It is worth noting that L-phenylalanine is involved in many pathways that are significantly changed in this study (table 3). L-phenylalanine is an essential amino acid and a precursor for the synthesis of antiviral and anticancer drugs as well as new sweeteners. It can also be a marker of inflammatory reactions and affect the body's immune response [33]. Here, the content of L-phenylalanine was significantly increased on day 7 , which means that the immune defenses in the mammary gland may have been effectively improved. Previous study [35] found that tryptophan and phenylalanine are used in the body to synthesize serotonin, L-DOPA derivatives and 5, 6, 7, 8-tetrahydrobiopterin (BH4), which compounds can affect the severity of inflammation. In the study of Smith ,[39], tyrosine and phenylalanine participated in the regulation of TLR4 signaling pathways, thereby influencing the degree of inflammation.

Phenylalanine also is part of the glycolytic and liposynthetic pathways.[40, 41]. It has been reported that phenylalanine deficiency can severely affect protein metabolism in the breast and compromise the health of the organ.[42]. Phenylalanine is an essential amino acid in milk and is also the precursor of tyrosine, which is one of the main amino acids in milk.[43]. We know that untreated bovine mastitis results in reduced milk fat and milk protein content; therefore, the increase in L-phenylalanine by $\mathrm{MCH}$ injection could be effective in restoring normal milk production during lactation.

The milk metabolites produced by $\mathrm{MCH}$ injection were found to have an intimate relationship with sphingolipid and glycerophospholipid metabolism. A previous study suggested that the sphingolipid metabolic pathways participate in a variety of immune-related signal transductions, inflammation and inflammatory disorders [29]. The sphingolipid metabolism-related pathways have many functions, such as regulating cell adhesion and cellular immunity, activating cancer repressors, regulating apoptosis, and modulating immune function and the inflammatory response [29] We speculate that this activity might be associated with the decrease in SCC caused by MCH injection. Furthermore, Cowart et al. [44] showed that an imbalance in the pathway for sphingolipid metabolism can cause ketosis, mastitis, and metritis in cows. It has been proved that sphingolipid metabolites, especially ceramide and sphingosine-1phosphate, can regulate a variety of biochemical processes important in immune, inflammation and inflammatory diseases[29]. In the present study, we found that MCHs could significantly regulate the sphingolipid metabolism-related pathways to improve inflammation; thus, it is reasonable to believe that MCHs could effectively treat bovine mastitis.

Despite the documented health-promoting properties of matrine and chitosan, scientific evidence for the efficacy of MCHs in dairy cows is limited. Our study affords many useful insights, but the effect of MCHs 
on immune regulation still requires further study. Another limitation relates to the mechanistic links involved in the observed significant changes in the microbiota and biomarker metabolites as a result of $\mathrm{MCH}$ injection that will need further exploration to be clearly understood. Previous studies suggested that the antimicrobial activity of chitosan can stimulate the innate immune response and hasten the involution process of the mammary gland[25]. It is not known whether matrine-chitosan complexes could be used in the circulation to regulate the immune system and inhibit inflammation, but our data indicate that future research in this area is strongly warranted.

\section{Conclusions}

Our data indicate that matrine-chitosan hydrogels significantly decrease the somatic cell counts and affect the structures of bacterial communities in the udder, especially the relative abundances of Corynebacterium_1, Aerococcus and Staphylococcus. These findings show significant changes in metabolites and metabolic pathways as a result of intramammary $\mathrm{MCH}$ infusion, and some of the resulting 74 milk metabolites may be used as indicators of the response to $\mathrm{MCH}$ treatment. The results from the milk metabolic pathway analysis are promising for the investigation of matrine-chitosan's antimicrobial and anti-inflammatory properties that are closely associated with sphingolipid and glycerophospholipid metabolism. These insights into the complex mechanisms and corresponding biological responses highlight the beneficial action of matrine-chitosan hydrogels and justify continued investigations to identify the immunoregulatory mechanisms for treatment of mastitis dairy cows.

\section{Methods}

\subsection{Preparation of matrine-loaded chitosan hydrogels}

Matrine (98\% purity) was purchased from Sigma-Aldrich Corp., St. Louis, MO, USA, and chitosan was purchased from Shanghai Sunny Biotech Co., Ltd., China. All solutions were prepared with nonpyrogenic products and materials under aseptic conditions in a laminar flow hood. The procedures were similar to those of Lanctôt et al. [25]. Matrine $(0.05 \mathrm{~g})$ and $4 \mathrm{~g}$ of chitosan were added to $95 \mathrm{~mL}$ of deionized water and heated at $37^{\circ} \mathrm{C}$ until the chitosan dissolved to obtain the matrine-loaded chitosan complexes. Ten $\mathrm{ml}$ of matrine-chitosan and $5 \mathrm{ml}$ of $4 \%$ hydroxyethyl cellulose (2:1 ratio) were put into disposable plastic dishes (60 mm diameter) and mixed thoroughly. The water used was nonpyrogenic with $<0.005$ endotoxin units/mL (Lonza, Walkersville, MD). For intramammary infusion, plastic syringes were filled with the $10 \mathrm{ml}$ of $\mathrm{MCH}$, sealed with a cap, and stored at room temperature.

\subsection{Animals and experimental design}

Animals selected in this study were provided by the Beijing Sunlow Livestock Dairy Farming Center (Beijing, China), our animal protocols were reviewed and accepted by the Animal Care Committee of Beijing University of Agriculture in concordance with the guidelines for the use of bovines in research studies of the SSTCC (The State Science and Technology Commission of the P.R. of China, 2017). The dairy farm owner was consent to collect samples from cows for the presnet study. Samplings of 
untreated milk ( $n=580)$ were received from commercial dairies in Beijing from June to August 2019 with the California mastitis rapid detection reagent (CMT). Six mid-lactation cows with high somatic cell counts (SCC, average 250,000 cells/ml) indicative of subclinical-mastitis were selected according to their milk yield and parity. There were few initial differences in milk yield $(27.2 \pm 1.8 \mathrm{~kg} / \mathrm{d})$, DIM $(114.6 \pm 7.5 \mathrm{~d})$, parity $(2.6 \pm 0.4)$, or body weight (BW; $670 \pm 24 \mathrm{~kg}$ ). The cows were fed a standard basal diet (table 4), had free access to water and were kept in a tie-stall barn. They received daily intramammary infusions of MCHs after each milking, twice daily, (7 AM and 7 PM) for 7 days. Milk samples were collected on the first day (D 1) and the last injection day (D 7) and totaled $15 \mathrm{ml}$ per animal, with about equal volumes from each lactating udder quarter. Somatic cell counts were determined using an automatic cell counter (DeLaval International AB, Tumba, Sweden), and the remaining milk was stored in liquid nitrogen for later analysis. After the period of experimental, all dairy cows were back to the herd of cows after the veterinary examination and ensure they were healthy.

\subsection{Bacterial DNA isolated, $16 \mathrm{~S}$ rRNA genes amplified and sequenced}

Bacterial DNA was isolated from milk using a Power Soil DNA isolation kit (Qiagen, U.K.) as previously stated by Tong et al.[14]. In brief, the DNA concentration and 260/280 ratio were measured with a NanoDrop 1000 spectrophotometer (Nanodrop Technologies, U.S.), and integrity was visualized by running aliquots on an agarose gel. The V1-V2 region of the 16S rRNA gene was PCR-amplified by (GeneAmp 9700, ABI, U.S.) using forward (5'-CGTATCGCCT-CCCTCGCGCCATCAG-3') and reverse (5'-CTATGCGCCTTGCCAGCCCGCTCAG-3') primers that incorporated adaptors and barcodes [14, 45]. Amplicons of about $450 \mathrm{bp}$ were selected and combined in equal concentrations [46], then electrophoresed and extracted from the gel with GeneJET (ThermoFisher, Waltham, MA, United States). Paired-end libraries were prepared by Majorbio Bio-Pharm (Shanghai, China). Bacterial ribosomal RNA genes were sequenced with Illumina HiSeq (Illumina, U.S.) for paired-end reads of three hundred base pairs. All raw sequence data were uploaded to NCBI (\#SRP254162).

\subsection{Analytical bioinformatics of milk microbiotas}

Analyses were conducted with FLASH version 1.2.11 and quantitative insights into microbial ecology (QIIME) version 1.9.1. These programs gave data similar to that published by Tong et al. [14]. Sequences were assigned to taxa by BLASTing the ribosomal database project (RDP) dbase using a $97 \%$ identity cutoff. Operational taxonomic units (OTUs) were normalized to relative abundance and bacterial

composition was determined by Majorbio I-Sanger. Within-sample diversity (a-diversity) was measured as bacterial community enrichment (ACE and Chao indices) and diversity (Shannon and Simpson indices) that were measured in a stochastic subset of the OTUs. Between-sample microbial diversity ( $\beta$-diversity) was determined by phylogenetically-based weighted UniFrac distances [47]. A more detailed picture of the diversity of the most abundant evolutionary clades in the bovine microbiota was obtained by filtering the OTUs to yield those with a relative abundance of $\geq 1 \%$ for at least one sample.

\subsection{Milk metabolome determination}


Milk samples were assessed by Majorbio Bio-Pharm using the LC-MS AB Sciex Triple TOF 5600TM (AB SCIEX, U.S.) according to published procedures [14] The liquid chromatography conditions were similar to those of a previous study [48]. Quality controls were run by combining milk samples and injecting them periodically during experimental measurements. The results were analyzed with XCMS (ver. 3.4.4). The retention times, $\mathrm{MZ}$, observations and peak intensities were normalized with Excel. The differentially expressed metabolites were analyzed with the public database (https://metlin.scripps.edu/) on the Majorbio I-Sanger platform (www.i-sanger.com) and the KEGG pathway software for differential metabolite profiles (www.metaboanalyst.ca/).

\subsection{Multivariate statistics}

Comparisons were validated by Student's $t$ and $p<0.05$ was defined as significant. Hierarchical clustering was conducted using the Bray-Curtis similarity index and the unweighted pair-group method with arithmetic averages. The SPSS software v.21.0 (IBM, Armonk, NY) was used. The a-diversity indices are given as mean $\pm S D$. A $p<0.05$ was considered statistically significant, and a $p<0.10$ suggested a trend. Principal component analysis and orthogonal partial least-squares-discriminant analysis (OPLS-DA) were carried out to show the metabolism changes among the experimental groups after mean centering and unit variance scaling. Parameters with variable importance in the projection (VIP) values $>1.0$ were allowed for group discriminant testing. Our OPLS-DA model was confirmed by 7-fold permutation testing. Significantly different metabolites among groups were assessed and identified by Wilcoxon rank-sum tests.

\section{Abbreviations}

MCHs: Matrine-chitosan hydrogels; PCA:Principal component analysis; OPLS-DA:Orthogonal partial least squares discriminant analysis; KEGG:Kyoto Encyclopedia of Genes and Genomes; M: metabolism; HD: human diseases; EIP: environmental information processing; OS: organismal systems; GIP: genetic information processing; SCC: somatic cell counts; CMT: California mastitis rapid detection reagent; RDP:Ribosomal database project; OTUs: Operational taxonomic units

\section{Declarations}

\section{Acknowledgements}

The authors thank the dairy barn staff for taking care of the cows, especially Kangkang Chu for providing technical assistance. The authors are grateful to Dandan He, from Shanghai Majorbio Bio-pharm Technology Co.,Ltd, for her assistant of the multivariate statistics analysis.

\section{Authors' contributions}

JJT, FXW and LSJ developed hypothesis, conceived the project and responsible for all data, figures and text. JJT, HZ, HN and FXW performed the experiments. FXW and HZ conducted data analysis. JJT and 
FXW wrote the manuscript. JJT, HZ, LSJ and BHX revised the paper. All authors carefully read the manuscript and agree to be held accountable for all aspects of the work.

\section{Funding}

This study was funded by the Project of the National Natural Science Foundation of China (Grant Nos. 31802091, 31702302 and 31772629) and the National Key Research and Development Plan (2016YFD0700205, 2016YFD0700201, and 2017YFD0701604). Jinjin Tong also thanks the China Postdoctoral Science Foundation and the Beijing Postdoctoral Science Foundation. Linshu Jiang thanks for the Beijing million Talents Project.

\section{Availability of data and materials}

The datasets used and/or analyzed during the current study are available from the corresponding author on reasonable request.

\section{Ethics approval and consent to participate}

The animal protocols were reviewed and accepted by the Animal Care Committee of Beijing University of Agriculture in concordance with the guidelines for the use of bovines in research studies of the SSTCC (The State Science and Technology Commission of the P.R. of China, 2017).

\section{Consent for publication}

Not Applicable.

\section{Conflicts of Interest}

The authors declare no conflicts of interest regarding this work

\section{Author details}

\section{References}

1. Derakhshani H, Plaizier JC, De Buck J, Barkema HW, Khafipour E: Association of bovine major histocompatibility complex (BoLA) gene polymorphism with colostrum and milk microbiota of dairy cows during the first week of lactation. MICROBIOME 2018, 6(1):203.

2. Gao J, Barkema HW, Zhang L, Liu G, Deng Z, Cai L, Shan R, Zhang S, Zou J, Kastelic JP, et al. Incidence of clinical mastitis and distribution of pathogens on large Chinese dairy farms. J DAIRY SCI. 2017;100(6):4797-806.

3. Lhermie G, Gröhn YT, Raboisson D. Addressing Antimicrobial Resistance: An Overview of Priority Actions to Prevent Suboptimal Antimicrobial Use in Food-Animal Production. FRONT MICROBIOL. 2017;7:2114. 
4. Ji X, Guo J, Liu Y, Lu A, Wang Z, Li Y, Yang S, Wang Q. Marine-Natural-Product Development: First Discovery of Nortopsentin Alkaloids as Novel Antiviral, Anti-phytopathogenic-Fungus, and Insecticidal Agents. J AGR FOOD CHEM. 2018;66(16):4062-72.

5. Feng F, Ma WW, Luo HX, Guan CP, Zhou XZ. Effect of matrine on reducing damage to bovine mammary epithelial cells induced by Staphylococcus aureus alpha-hemolysin. POL J VET SCI. 2018;21(2):409-13.

6. Kaiyue S, Pengyu, Yang R, Zhao, Yuting, Bai, Zijiao, Guo: Matrine Attenuates D-Galactose-Induced Aging-Related Behavior in Mice via Inhibition of Cellular Senescence and Oxidative Stress. Oxidative Medicine \& Cellular Longevity 2018.

7. Zhou W, Zhao J, Pan X, Sun Y, Tian X. Matrine alleviates lipopolysaccharide-induced intestinal inflammation and oxidative stress via CCR7 signal. Oncotarget. 2017;8(7):11621.

8. Hua Z, Jinjin T, Zhaonan Z, Linshu J. Research Progress on Regulatory Effect and Mechanism of Chitooligosaccharides on Immunity and Inflammation in Animals. Chinese Journal of Animal Nutrition 2019.

9. Sun M, Tong J, Zhang H, Yang D, Zhang J, Jiang L, Xiong B. Chitosan: Biochemical Functions and Application in Dairy Cows. Chinese Journal of Animal Nutrition 2018.

10. Chenite A, Chaput C, Wang D, Combes C, Selmani A. Novel Injectable Neutral Solutions of Chitosan form Biodegradable Gels in situ. BIOMATERIALS. 2000;21(21):2155-61.

11. Oikonomou G, Bicalho ML, Meira E, Rossi RE, Foditsch C, Machado VS, Teixeira AGV, Santisteban C, Schukken YH, Bicalho RC. Microbiota of cow's milk; distinguishing healthy, sub-clinically and clinically diseased quarters. PLOS ONE. 2014;9(1):e85904.

12. Hélène F, Lucie R, Aurélie, Nicolas, Damien S, Bouchard J. Bovine Teat Microbiome Analysis Revealed Reduced Alpha Diversity and Significant Changes in Taxonomic Profiles in Quarters with a History of Mastitis. FRONT MICROBIOL 2016.

13. Sun HZ, Kai S, Wu XH, Xue MY, Wei ZH, Liu JX, Liu HY. Lactation-related metabolic mechanism investigated based on mammary gland metabolomics and 4 biofluids' metabolomics relationships in dairy cows. BMC GENOMICS. 2017;18(1):936.

14. Tong J, Zhang H, Zhang Y, Xiong B, Jiang L. Microbiome and Metabolome Analyses of Milk From Dairy Cows With Subclinical Streptococcus agalactiae Mastitis-Potential Biomarkers. FRONT MICROBIOL. 2019;10:2547.

15. Junza A, Saurina J, Barrón D, Minguillón C. Metabolic profile modifications in milk after enrofloxacin administration studied by liquid chromatography coupled with high resolution mass spectrometry. $J$ CHROMATOGR A. 2016;1460(Complete):92-9.

16. Mohammed R, Brink GE, Stevenson DM, Neumann AP, Beauchemin KA, Suen G, Weimer PJ. Bacterial communities in the rumen of Holstein heifers differ when fed orchardgrass as pasture vs. hay. FRONT MICROBIOL 2014, 5.

17. Schokker D, Veninga G, Vastenhouw SA, Bossers A, Smits MA. Early life microbial colonization of the gut and intestinal development differ between genetically divergent broiler lines. BMC GENOMICS. 
2015;16(1):418.

18. Barbosa J, Junza, Alexandra, Barron. Dolores, Blanco, Guillermo, Segarra, David: Wildlife contamination with fluoroquinolones from livestock: Widespread occurrence of enrofloxacin and marbofloxacin in vultures. Chemosphere Environmental Toxicology \& Risk Assessment 2016.

19. Oliveira L, Ruegg PL. Treatments of clinical mastitis occurring in cows on 51 large dairy herds in Wisconsin. J DAIRY SCI. 2014;97(9):5426-36.

20. Bhat R, Amin I, Ali A, Mir B, Bashir S, Bashir N, Ahmad SB, Baba O, Muzamil S, Reshi P, et al: Association of somatic cell count and hs-CRP acute phase protein with bovine subclinical mastitis in Holstein Frisian cross bred cattle of Kashmir. 2018.

21. Vanderhaeghen W, Piepers S, Leroy F, Coillie EV, Haesebrouck F, Vliegher SD. Invited review: Effect, persistence, and virulence of coagulase-negative Staphylococcus species associated with ruminant udder health. J DAIRY SCI. 2014;97(9):5275-93.

22. Microbiota of Cow's Milk; Distinguishing Healthy, Sub-Clinically and Clinically Diseased Quarters. PLOS ONE 2014, 9.

23. Barkema HW, Schukken YH, Zadoks RN. Invited Review: The Role of Cow, Pathogen, and Treatment Regimen in the Therapeutic Success of Bovine Staphylococcus aureus Mastitis. J DAIRY SCI. 2006;89(6):1877-95.

24. Rainard P. Mammary microbiota of dairy ruminants: Fact or fiction? VET RES. 2017;48(1):25.

25. Lanct TS, Fustier P, Taherian AR, Bisakowski B, Zhao X, Lacasse P. Effect of intramammary infusion of chitosan hydrogels at drying-off on bovine mammary gland involution. J DAIRY SCI. 2017;100(3):2269-81.

26. Catozzi C, Bonastre AS, Francino O, Lecchi C, Ceciliani F. The microbiota of water buffalo milk during mastitis. PLOS ONE. 2017;12(9):e184710.

27. Bhatt, Sima K. BACTERIOLOGICAL PROFILE AND ANTIBIOGRAM OF NEONATAL SEPTICEMIA. National Journal of Community Medicine 2012.

28. Zhao S, Zhao J, Bu D, Sun P, Wang J, Dong Z. Metabolomics analysis reveals large effect of roughage types on rumen microbial metabolic profile in dairy cows. LETT APPL MICROBIOL. 2014;59(1):79-85.

29. Maceyka M, Spiegel S. Sphingolipid metabolites in inflammatory disease. NATURE. 2014;510(7503):58-67.

30. Thomas FC, Mudaliar M, Tassi R, Mcneilly TN, Burchmore R, Burgess K, Herzyk P, Zadoks RN, Eckersall PD: Mastitomics, the integrated omics of bovine milk in an experimental model of Streptococcus uberis mastitis: 3. Untargeted metabolomics. MOL BIOSYST 2016, 12(9):2762-2769.

31. Li N, Sancak Y, Frasor J, Atilla-Gokcumen GE. A protective role for triacylglycerols during apoptosis. BIOCHEMISTRY-US 2017:7b-975b.

32. Kagan VE, Mao G, Feng Q, Angeli JPF, Bayır H. Oxidized arachidonic and adrenic PEs navigate cells to ferroptosis. NAT CHEM BIOL 2016, 13(1). 
33. Turk $\mathrm{R}$. The role of oxidative stress and inflammatory response in the pathogenesis of mastitis in dairy cows. MLJEKARSTVO 2017:91-101.

34. Li L, Wang H, Nie X, Jiang W, Zhang Y. Sodium butyrate ameliorates lipopolysaccharide-induced cow mammary epithelial cells from oxidative stress damage and apoptosis. J CELL BIOCHEM 2018.

35. Strasser B, Sperner-Unterweger B, Fuchs D, Gostner JM: Mechanisms of Inflammation-Associated Depression: Immune Influences on Tryptophan and Phenylalanine Metabolisms: Curr Top Behav Neurosci; 2016.

36. Gao X, Guo S, Zhang S, Liu A, Shi L, Zhang Y. Matrine attenuates endoplasmic reticulum stress and mitochondrion dysfunction in nonalcoholic fatty liver disease by regulating SERCA pathway. $J$ TRANSL MED. 2018;16(1):319.

37. Gao D, Wang Y, Xie W, Yang T, Jiang Y, Guo Y, Guan J, Liu H. Metabolomics study on the antitumor effect of marine natural compound flexibilide in HCT-116 colon cancer cell line. Journal of Chromatography B 2016:S2078752766.

38. X G. S G, S Z, A L, L S, Y Z: Matrine attenuates endoplasmic reticulum stress and mitochondrion dysfunction in nonalcoholic fatty liver disease by regulating SERCA pathway. J TRANSL MED. 2018;16(1):319.

39. Van Slyke Paul LST, Nina J, J. DD CJM. Tie2 signalling through Erk1/2 regulates TLR4 driven inflammation. CELL SIGNAL 2018:S663876812.

40. Waisbren SE, Noel K, Fahrbach K, Cella C, Frame D, Dorenbaum A, Levy H. Phenylalanine blood levels and clinical outcomes in phenylketonuria: A systematic literature review and meta-analysis. Molecular Genetics Metabolism. 2007;92(1-2):70.

41. Obianom C, Romanazzi G, Sivakumar D. Effects of chitosan treatment on avocado postharvest diseases and expression of phenylalanine ammonia-lyase, chitinase and lipoxygenase genes. Postharvest Biology Technology. 2019;147:214-21.

42. Shibata K, Shiotani M, Onodera M, Suzuki T. Changes in Nicotinamide Metabolism by One Amino Acid Deficiency. (I) Threonine-, Tryptophan-, Aspartic Acid-, Lysine-, Leucine-, or Methionine-free Diet. Journal of the Agricultural Chemical Society of Japan. 1992;56(5):783-7.

43. Rezaei R, Wu Z, Hou Y, Bazer FW, Wu G. Amino acids and mammary gland development: nutritional implications for milk production and neonatal growth. Journal of Animal Science Biotechnology. 2016;7(1):20.

44. Brice SE, Cowart LA. Sphingolipid metabolism and analysis in metabolic disease. Oxygen Transport to Tissue XXXIII. 2011;721(721):1.

45. Oikonomou G, Trojacanec P, Ganda EK, Bicalho MLS, Bicalho RC. Association of digital cushion thickness with sole temperature measured with the use of infrared thermography. J DAIRY SCI. 2014;97(7):4208-15.

46. Li Y, Hu X, Shuang Y, Zhou J, Lei Q, Sun X, Fan M, Xu S, Muha C, Zhang M. Comparison Between the Fecal Bacterial Microbiota of Healthy and Diarrheic Captive Musk Deer. FRONT MICROBIOL. 2018;9:300. 
47. Lozupone C, Knight R. UniFrac: a New Phylogenetic Method for Comparing Microbial Communities. Applied Environmental Microbiology. 2006;71(12):8228-35.

48. Wang X, Sun G, Feng T, Zhang J, Huang X, Wang T, Xie Z, Chu X, Yang J, Wang H, et al. Sodium oligomannate therapeutically remodels gut microbiota and suppresses gut bacterial amino acidsshaped neuroinflammation to inhibit Alzheimer's disease progression. CELL RES. 2019;29(10):787803.

\section{Tables}

Table 1. Alpha diversity indices for milk microbiota

\begin{tabular}{llll}
\hline Item & D 1 & D 7 & p values \\
\hline ACE & $1769.86 \pm 189.07$ & $1211.79 \pm 196.29$ & 0.08 \\
& & & \\
\hline Chao & $1675.43 \pm 201.72$ & $1176.47 \pm 196.24$ & 0.11 \\
& & & \\
\hline Simpson & $0.47 \pm 0.09$ & $0.19 \pm 0.07$ & $0.05^{*}$ \\
\hline Shannon & $3.35 \pm 0.52$ & $1.92 \pm 0.36$ & 0.06 \\
\hline Coverage & $0.99 \pm 0.01$ & $0.99 \pm 0.02$ & 0.57 \\
\hline
\end{tabular}

Table 2. Milk metabolites on day 1 compared to day 7 after MCH treatment 


\begin{tabular}{|c|c|c|c|c|c|c|c|}
\hline Metabolite & $\mathrm{M} / \mathrm{Z}$ & $\begin{array}{l}\text { Retention } \\
\text { time }\end{array}$ & $\begin{array}{l}\text { Mass } \\
\text { Error }\end{array}$ & VIP & $\mathrm{FC}(\mathrm{d} 1 / \mathrm{d} 7)$ & $\begin{array}{l}\mathrm{P} \\
\text { value }\end{array}$ & Tendency \\
\hline \multicolumn{8}{|l|}{ Steroids and steroid derivatives } \\
\hline 12b-Hydroxy-5b-cholanoic acid & 418.35 & 9.43 & 1.12 & 4.42 & 0.48 & 0.01 & $\uparrow$ \\
\hline Pregnanetriolone & 349.24 & 8.8 & -0.06 & 1.79 & 0.43 & 0.026 & $\uparrow$ \\
\hline Aginoside progenin & 814.46 & 2.51 & -2.8 & 1.02 & 0.04 & $\square 0.01$ & $\uparrow$ \\
\hline \multicolumn{8}{|l|}{ Sphingolipids } \\
\hline SM(d18:0/24:1(15Z)) & 859.7 & 13.36 & 5.06 & 1.58 & 3208275862 & $\square 0.01$ & $\downarrow$ \\
\hline $\mathrm{SM}(\mathrm{d} 18: 1 / 23: 0)$ & 845.68 & 12.95 & 5.27 & 2.26 & 11748.6 & $\square 0.01$ & $\downarrow$ \\
\hline SM(d18:1/22:1(13Z)) & 829.65 & 11.92 & 4.59 & 1.72 & 369.77 & $\square 0.01$ & $\downarrow$ \\
\hline SM(d18:0/22:1(13Z)) & 831.66 & 12.73 & 4.91 & 4.9 & 212.45 & $\square 0.01$ & $\downarrow$ \\
\hline Glucosylceramide (d18:1/16:0) & 744.57 & 11 & 3.44 & 1.89 & 34.01 & $\square 0.01$ & $\downarrow$ \\
\hline Galactosylceramide (d18:1/14:0) & 706.51 & 10.5 & 3.07 & 1.29 & 27.94 & $\square 0.01$ & $\downarrow$ \\
\hline Glucosylceramide (d18:1/25:0) & 790.69 & 14.01 & -0.89 & 3.78 & 21.09 & $\square 0.01$ & $\downarrow$ \\
\hline stearoyl sphingomyelin & 775.6 & 11.48 & 4.77 & 1.32 & 19.61 & $\square 0.01$ & $\downarrow$ \\
\hline N-hexadecanoylsphinganine-1-phosphocholine & 749.58 & 11.16 & 2.57 & 2.76 & 16.13 & $\square 0.01$ & $\downarrow$ \\
\hline Galabiosylceramide (d18:1/16:0) & 906.62 & 10.86 & 5.8 & 1.9 & 9.04 & $\square 0.01$ & $\downarrow$ \\
\hline $\mathrm{SM}(\mathrm{d} 18: 0 / 14: 0)$ & 721.55 & 10.62 & 3.35 & 2.94 & 7.99 & $\square 0.01$ & $\downarrow$ \\
\hline $\mathrm{SM}(\mathrm{d} 18: 0 / 16: 1(9 \mathrm{Z}))$ & 747.57 & 10.92 & 4.52 & 6.89 & 3.62 & $\square 0.01$ & $\downarrow$ \\
\hline N-Glycoloylganglioside GM2 & 708.61 & 12.75 & -0.87 & 9.56 & 2.38 & $\square 0.01$ & $\downarrow$ \\
\hline $\mathrm{SM}(\mathrm{d} 18: 1 / 14: 0)$ & 719.54 & 10.42 & 3.7 & 5.12 & 2.34 & $\square 0.01$ & $\downarrow$ \\
\hline Araliacerebroside & 776.55 & 10.75 & -6.85 & 1.95 & 1.49 & $\square 0.01$ & $\downarrow$ \\
\hline Ganglioside GM1 (d18:1/18:1(11Z)) & 772.43 & 4.33 & -7.63 & 1 & 0.28 & 0.02 & $\uparrow$ \\
\hline nLc6Cer & 696.82 & 3.25 & -1.78 & 2.36 & 0.09 & $\square 0.01$ & $\uparrow$ \\
\hline \multicolumn{8}{|l|}{ Prenol lipids } \\
\hline Hoduloside VI & 819.43 & 2.96 & -3.62 & 1.42 & 0.05 & 0.01 & $\uparrow$ \\
\hline \multicolumn{8}{|l|}{ Oxanes } \\
\hline D-1,5-Anhydrofructose & 325.11 & 0.78 & -0.78 & 1.1 & 0.78 & $\square 0.01$ & $\uparrow$ \\
\hline \multicolumn{8}{|l|}{ Organooxygen compounds } \\
\hline $\begin{array}{l}\text { 3,4,5-trihydroxy-6-(2-hydroxyethoxy)oxane-2-carboxylic } \\
\text { acid }\end{array}$ & 475.13 & 0.7 & -1.46 & 1.13 & 2.62 & $\square 0.01$ & $\downarrow$ \\
\hline N6-Galacturonyl-L-lysine & 357.11 & 2.4 & 8.9 & 2.54 & 1.56 & 0.02 & $\downarrow$ \\
\hline Lactulose & 365.11 & 0.62 & 1.53 & 6.7 & 0.56 & $\square 0.01$ & $\uparrow$ \\
\hline \multicolumn{8}{|l|}{ Macrolides and analogues } \\
\hline Pectenotoxin 7 & 909.37 & 2.45 & -1.88 & 1.95 & 0.1 & $\square 0.01$ & $\uparrow$ \\
\hline 31-O-Demethyltacrolimus & 810.44 & 0.75 & -1.37 & 1.15 & 0.03 & $\square 0.01$ & $\uparrow$ \\
\hline
\end{tabular}


Indoles and derivatives

Indole-3-carboxilic acid-O-sulphate

240

2.27

Hydroxy acids and derivatives

2-Hydroxy-3-methoxyestrone

$442.35 \quad 9.08$

1.05

$2.9 \quad 0.43$

$0.01 \uparrow \uparrow$

Glycerophospholipids

\begin{tabular}{|c|c|c|c|c|c|c|c|}
\hline $\mathrm{PE}(15: 0 / 22: 0)$ & 806.6 & 11.84 & 5.06 & 2.5 & 48.31 & $\square 0.01$ & $\downarrow$ \\
\hline $\operatorname{PE}(14: 0 / 22: 0)$ & 792.58 & 11.45 & 8.49 & 1.14 & 15.03 & $\square 0.01$ & $\downarrow$ \\
\hline $\operatorname{PS}(\operatorname{DiMe}(11,3) / \operatorname{MonoMe}(11,5))$ & 880.54 & 12.16 & 2.74 & 1.21 & 8.01 & $\square 0.01$ & $\downarrow$ \\
\hline PE(15:0/24:1(15Z)) & 832.61 & 11.77 & 5.01 & 4.77 & 6.11 & $\square 0.01$ & $\downarrow$ \\
\hline lysoPC(6:0) & 400.17 & 3.01 & -1.21 & 2.04 & 5.51 & $\square 0.01$ & $\downarrow$ \\
\hline $\mathrm{PE}(16: 0 / 16: 0)$ & 736.52 & 10.42 & 2.96 & 2.16 & 2.26 & $\square 0.01$ & $\downarrow$ \\
\hline PS(18:0/18:1(9Z)) & 810.53 & 11.5 & 5.99 & 2.68 & 2.12 & $\square 0.01$ & $\downarrow$ \\
\hline PE(18:2(9Z,12Z)/18:1(11Z)) & 786.53 & 12.04 & 4.76 & 7.92 & 1.99 & $\square 0.01$ & $\downarrow$ \\
\hline $\operatorname{PE}(15: 0 / 16: 0)$ & 722.5 & 10.33 & 5.04 & 3.2 & 1.85 & $\square 0.01$ & $\downarrow$ \\
\hline $\mathrm{PE}(15: 0 / 14: 0)$ & 694.47 & 9.94 & 3.99 & 1.33 & 1.72 & $\square 0.01$ & $\downarrow$ \\
\hline $\mathrm{PE}(15: 0 / 22: 1(13 Z))$ & 804.58 & 11.24 & 5.01 & 4 & 1.67 & $\square 0.01$ & $\downarrow$ \\
\hline PS(16:0/18:0) & 784.52 & 11.45 & 7.51 & 3.58 & 1.63 & $\square 0.01$ & $\downarrow$ \\
\hline LysoPE(0:0/18:2(9Z,12Z)) & 476.28 & 7.75 & -0.17 & 1.66 & 1.53 & 0.05 & $\downarrow$ \\
\hline $\mathrm{PE}(18: 1(9 \mathrm{Z}) / 16: 0)$ & 718.54 & 11.44 & 0.48 & 1.83 & 1.5 & 0.03 & $\downarrow$ \\
\hline $\mathrm{PE}(15: 0 / 20: 2(11 \mathrm{Z}, 14 \mathrm{Z}))$ & 774.53 & 10.37 & 4.68 & 1.03 & 1.39 & 0.02 & $\downarrow$ \\
\hline LysoPE(16:0/0:0) & 452.28 & 8.32 & 0.26 & 1.84 & 1.36 & 0.01 & $\downarrow$ \\
\hline PS(15:0/24:1(15Z)) & 830.6 & 11.21 & 4.84 & 1.71 & 1.3 & 0.02 & $\downarrow$ \\
\hline LysoPC(18:1(9Z)) & 566.35 & 8.31 & 0.06 & 3.94 & 0.68 & 0.01 & $\uparrow$ \\
\hline LysoPC (18:2(9Z,12Z)) & 564.33 & 7.79 & -1.16 & 5.06 & 0.56 & 0.01 & $\uparrow$ \\
\hline LysoPC(16:1(9Z)/0:0) & 538.32 & 7.58 & 1.76 & 1.12 & 0.55 & 0.02 & $\uparrow$ \\
\hline LysoPE(20:3(11Z,14Z,17Z)/0:0) & 502.29 & 8.11 & 0.14 & 1.65 & 0.5 & 0.02 & $\uparrow$ \\
\hline $\mathrm{PC}(18: 2(9 \mathrm{Z}, 12 \mathrm{Z}) / \mathrm{P}-18: 0)$ & 814.6 & 11.07 & 4.43 & 1.18 & 0.47 & 0.01 & $\uparrow$ \\
\hline LysoPE(20:4(5Z,8Z,11Z,14Z)/0:0) & 500.28 & 7.8 & -0.15 & 3.45 & 0.41 & $\square 0.01$ & $\uparrow$ \\
\hline LysoPE(18:2(9Z,12Z)/0:0) & 478.29 & 7.77 & 2 & 2.75 & 0.35 & $\square 0.01$ & $\uparrow$ \\
\hline LysoPC (18:1(11Z)) & 522.36 & 8.2 & 0.69 & 4.22 & 0.31 & $\square 0.01$ & $\uparrow$ \\
\hline LysoPE(0:0/22:5(7Z,10Z,13Z,16Z,19Z)) & 562.27 & 5.39 & -0.86 & 1.06 & 0.3 & $\square 0.01$ & $\uparrow$ \\
\hline 1-Linoleoylglycerophosphocholine & 520.34 & 7.69 & 0.61 & 4.27 & 0.17 & 0.01 & $\uparrow$ \\
\hline 1-(8Z,11Z,14Z-eicosatrienoyl)-glycero-3-phosphate & 502.29 & 7.7 & -0.01 & 1.27 & 0.14 & $\square 0.01$ & $\uparrow$ \\
\hline LysoPC (P-18:0) & 552.37 & 8.59 & 0.37 & 1.31 & 0.09 & $\square 0.01$ & $\uparrow$ \\
\hline TG(16:1(9Z)/16:1(9Z)/16:1(9Z)) & 845.68 & 13.12 & -9.94 & 3.56 & 1607.82 & $\square 0.01$ & $\downarrow$ \\
\hline
\end{tabular}


Flavonoids

\begin{tabular}{|c|c|c|c|c|c|c|c|}
\hline Menthoside & 723.2 & 0.7 & 4.79 & 4.57 & 2.69 & $\square 0.01$ & $\downarrow$ \\
\hline Isoscoparin 2"'-(6-(E)-ferulylglucoside) & 781.2 & 0.84 & -0.3 & 2.37 & 1.31 & 0.02 & $\downarrow$ \\
\hline Kaempferol 3-(2"'-rhamnosylgalactoside) 7-rhamnoside & 777.16 & 0.7 & -1.93 & 4.24 & 0.49 & $\square 0.01$ & $\uparrow$ \\
\hline Licorice glycoside C1 & 765.18 & 0.64 & 0.82 & 2.27 & 0.19 & $\square 0.01$ & $\uparrow$ \\
\hline \multicolumn{8}{|l|}{ Fatty Acyls } \\
\hline cis-Uvariamicin IB & 627.48 & 11.55 & 2.27 & 1.32 & 1268.67 & $\square 0.01$ & $\downarrow$ \\
\hline 3,4-Dimethyl-5-pentyl-2-furanpentadecanoic acid & 857.68 & 12.57 & -9.88 & 1.54 & 130.24 & $\square 0.01$ & $\downarrow$ \\
\hline 15-hydroxyicosanoic acid & 698.63 & 13.78 & -0.76 & 4.76 & 11.33 & $\square 0.01$ & $\downarrow$ \\
\hline 2-hydroxyhexadecanoic acid & 271.23 & 8.73 & 0.84 & 2.08 & 0.47 & 0.01 & $\uparrow$ \\
\hline 3-hydroxyhexadecanoyl carnitine & 416.34 & 8.99 & 2.02 & 2.15 & 0.43 & 0.01 & $\uparrow$ \\
\hline Aminocaproic acid & 132.1 & 1.17 & 0.75 & 1.42 & 0.04 & $\square 0.01$ & $\uparrow$ \\
\hline \multicolumn{8}{|l|}{ Carboxylic acids and derivatives } \\
\hline Tyrosyl-Isoleucine & 293.15 & 2.31 & -0.33 & 1.53 & 0.22 & $\square 0.01$ & $\uparrow$ \\
\hline L-Phenylalanine & 164.07 & 1.71 & 0.73 & 1.03 & 0.09 & $\square 0.01$ & $\uparrow$ \\
\hline \multicolumn{8}{|l|}{ Benzene and substituted derivatives } \\
\hline fluvoxamino acid & 360.15 & 0.86 & -8.35 & 2.72 & 0.53 & $\square 0.01$ & $\uparrow$ \\
\hline
\end{tabular}

Table 3. Differences in metabolites enriched from specific pathways in milk of dairy cows receiving $\mathrm{MCH}$ infusions (7 days). 
Metabolic Pathway

Metabolic pathways

(14)

Sphingolipid

metabolism (10)

Sphingolipid

signaling pathway (6)

Glycerophospholipid

metabolism (6)

ABC transporters (2)

2-Oxocarboxylic acid

metabolism (1)

Aminoacyl-tRNA

biosynthesis (1)

Amoebiasis (1)

Biosynthesis of

amino acids (1)

Carbohydrate

digestion and

absorption (1)

Glycine, serine and

threonine

metabolism (1)

Leishmaniasis (1)

Linoleic acid

metabolism (1)

Mineral absorption

(1)

Phenylalanine

metabolism (1)

Phenylalanine,

tyrosine and

tryptophan

biosynthesis (1)

Protein digestion and

absorption (1)

Starch and sucrose

metabolism (1)

Systemic lupus

erythematosus (1)

Taste transduction

(1)
Metabolite

Maltose;SM(d18:1/22:1(13Z));SM(d18:0/22:1(13Z));Aminocaproic acid;9R, 10S-EpOME;Glucosylceramide (d18:1/16:0);LPhenylalanine;SM(d18:0/16:1(9Z));Glucosylceramide

(d18:1/25:0);SM(d18:0/24:1(15Z));SM(d18:1/23:0);SM(d18:0/14:0);PS(18:0/18:1(9Z));Galactosylceramide (d18:1/14:0)

Galabiosylceramide (d18:1/16:0);SM(d18:1/22:1(13Z));SM(d18:0/22:1(13Z));Glucosylceramide

(d18:1/16:0);SM(d18:0/16:1(9Z));Glucosylceramide (d18:1/25:0);SM(d18:0/24:1(15Z));Galactosylceramide

(d18:1/14:0);SM(d18:0/14:0);SM(d18:1/23:0)

SM(d18:1/22:1(13Z));SM(d18:0/22:1(13Z));SM(d18:0/16:1(9Z));SM(d18:0/24:1(15Z));SM(d18:0/14:0);SM(d18:1/23:0) LysoPC(P-18:0);LysoPC(18:1(11Z));LysoPC(18:1(9Z));PS(18:0/18:1(9Z));LysoPC(16:1(9Z)/0:0);LysoPC (20:4(5Z, 8Z, 11Z, 14Z))

L-Phenylalanine; Maltose

L-Phenylalanine

L-Phenylalanine

PS(18:0/18:1(9Z))

L-Phenylalanine

Maltose

PS(18:0/18:1(9Z))

PS(18:0/18:1(9Z))

9R, 10S-EpOME

L-Phenylalanine

L-Phenylalanine

L-Phenylalanine

L-Phenylalanine

Maltose

PS(18:0/18:1(9Z))

Maltose

Table 4. Components and nutritional analysis of the total mixed rations (dry matter basis) 


\begin{tabular}{ll}
\hline Items & Content \\
\hline Alfalfa hay & 13.34 \\
Leymus chinensis & 11.20 \\
Corn & 15.73 \\
Whole cottonseed & 3.19 \\
Maize silage & 28.57 \\
DDGS & 2.99 \\
Steam-flaked corn & 7.16 \\
Soybean meal & 11.53 \\
Cottonseed meal & 3.87 \\
Premix1) & 1.96 \\
NaCl & 0.46 \\
Total & 100.00 \\
Nutrient levels & \\
NEL/(MJ/kg)2) & 7.26 \\
EE & 4.97 \\
CP & 17.35 \\
Neutral Washing Fiber NDF & 30.8 \\
ADF & 16.5 \\
Ca & 0.74 \\
P & 0.41 \\
\hline
\end{tabular}

Figures 


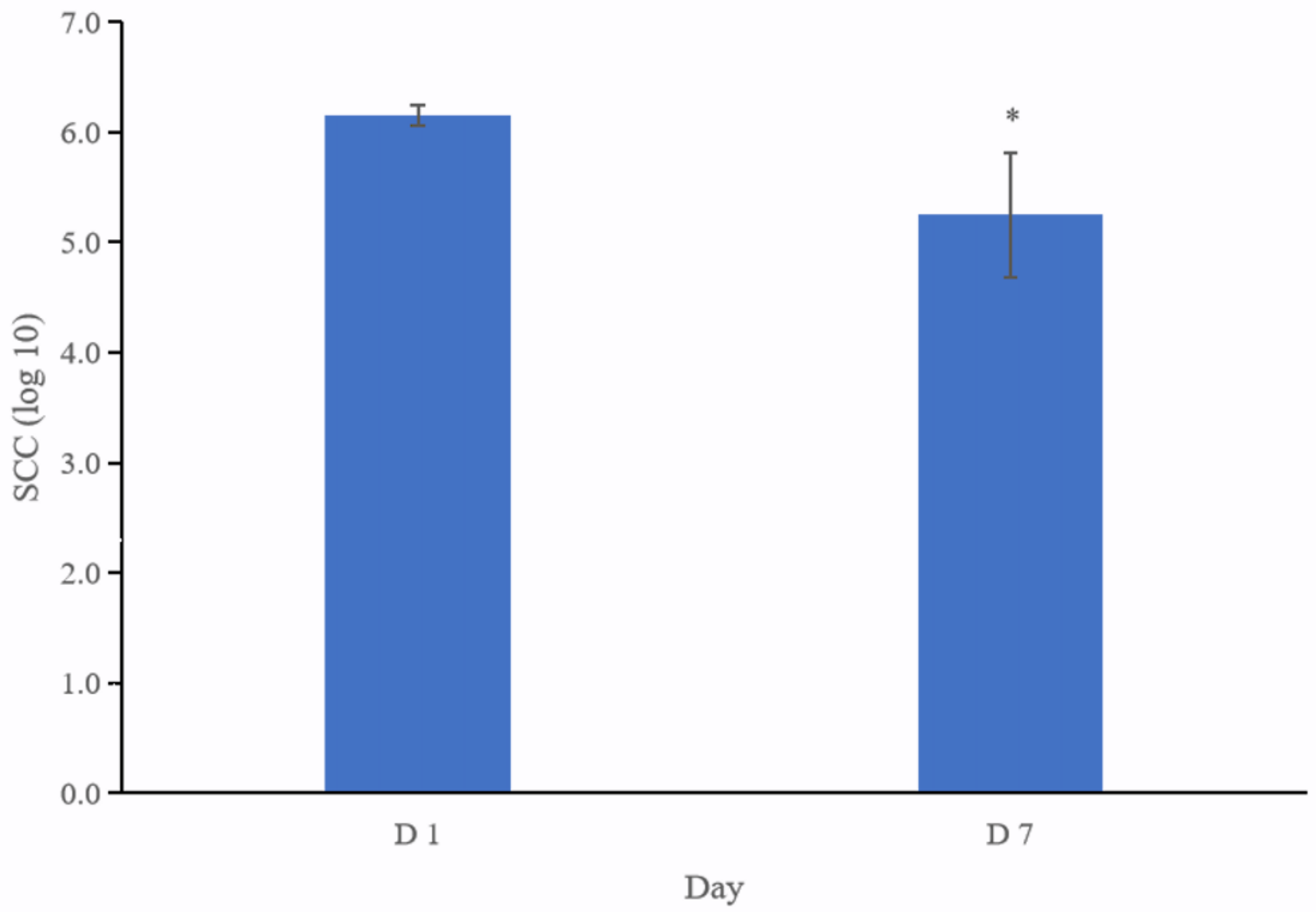

Figure 1

Somatic cell counts in milk from dairy cows after intramammary treatment with matrine-loaded chitosan hydrogels. 
PCA on OTU level

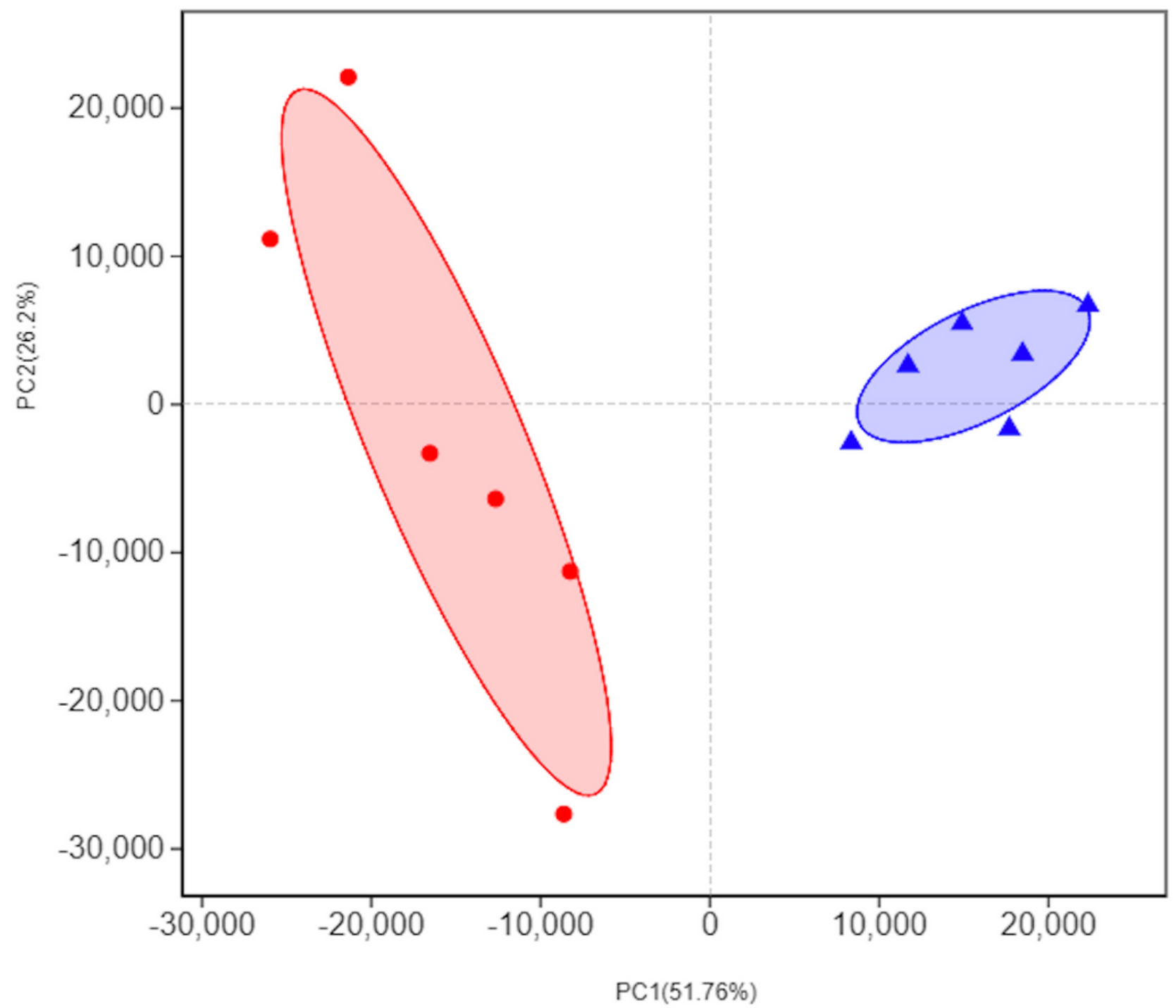

Figure 2

PCA of milk microbial communities from cows with subclinical mastitis treated with intramammary matrine-chitosan hydrogels from day 1 to day $7(n=6)$. 


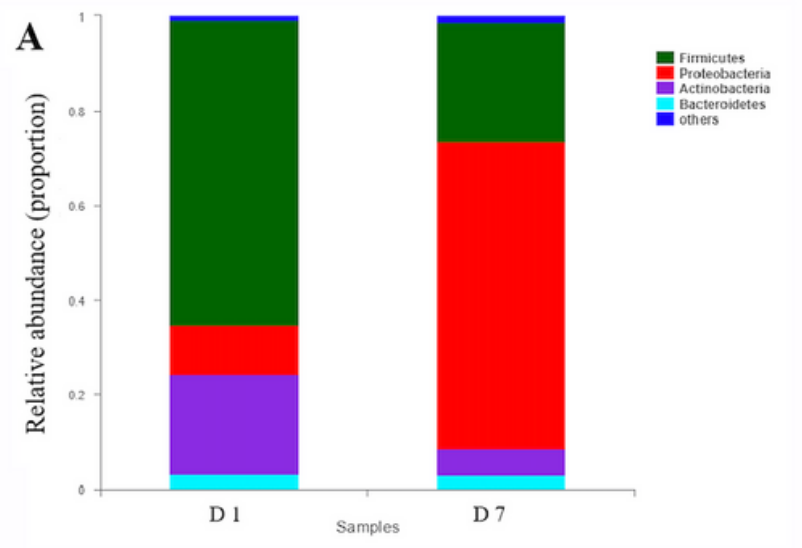

B

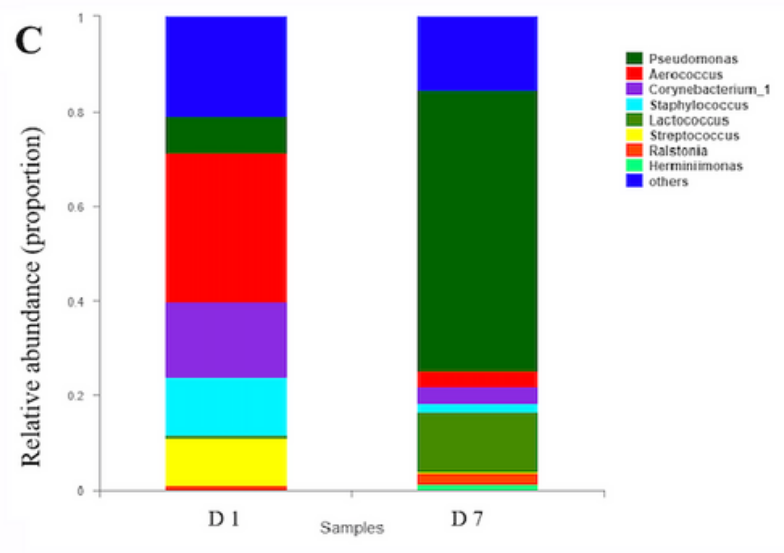

D

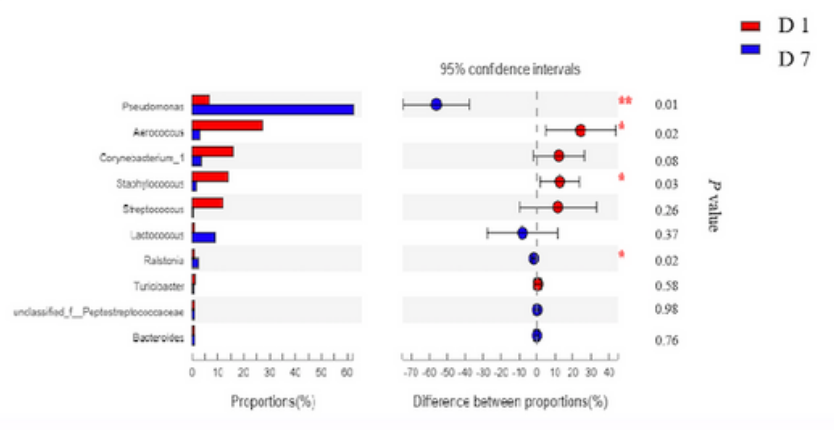

\section{Figure 3}

Classification of the bacterial community composition in milk samples on day 1 and day $7, n=6$. (A) Phylum level. (B) Relative abundance of the main bacterial phyla. (C) Differences in genera. (D) Differences in relative abundance of the main bacterial genera. The extended error bar plot was created with STAMP (Version v.2.1.3). Welch's two-sided test was used, and Welch's inverted test yielded 0.95.
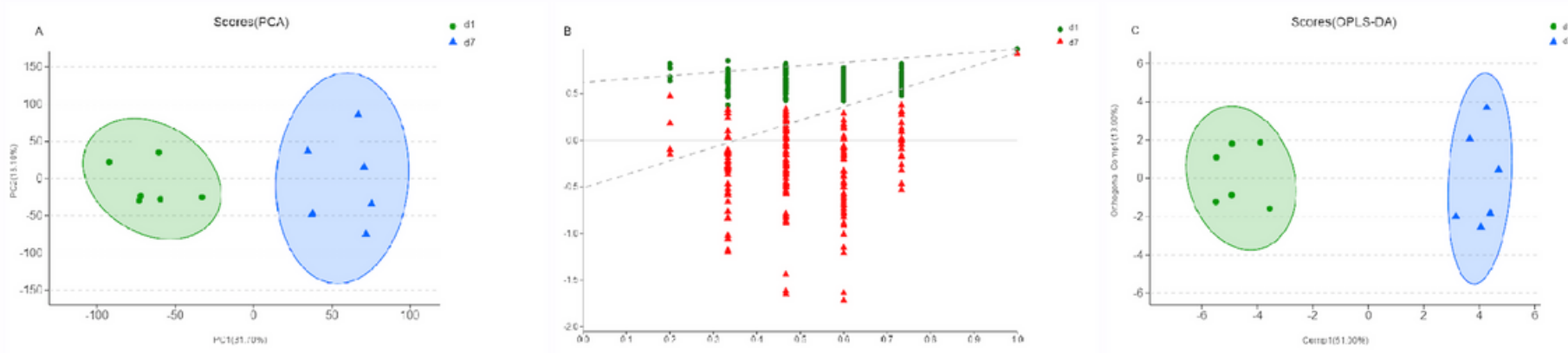

\section{Figure 4}

Principal component analysis score plot (A), permutation test plot (B) and orthogonal partial least squares discriminant analysis (OPLS-DA) (C) for days 1 and 7 based on milk metabolite profiles. The 
variation in the principal components is indicated on the axes. Each spot is one sample, and the days are shown as green circles for day 1 and blue triangles for day 7 .

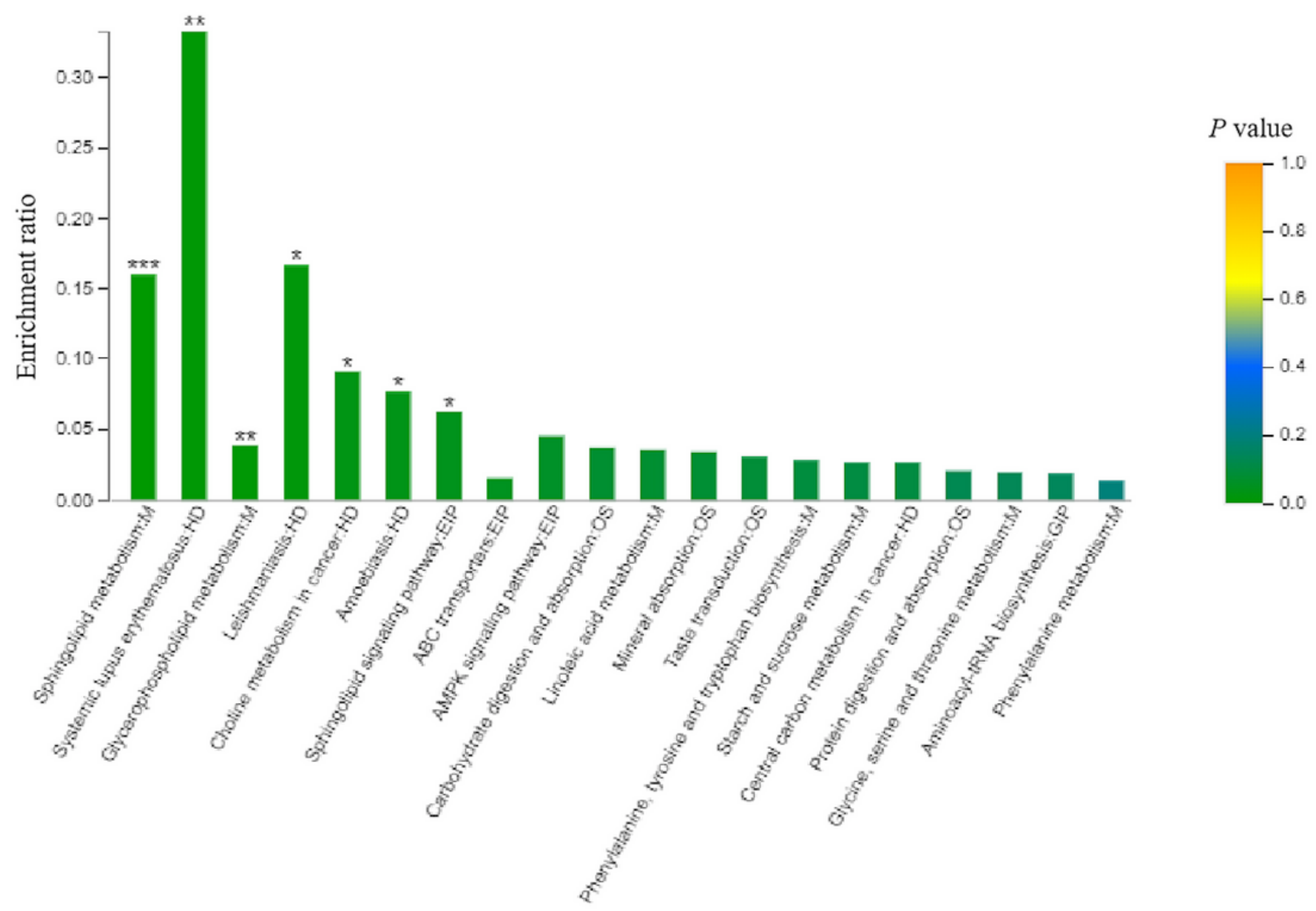

Figure 5

Metabolic pathway enrichment analysis between day 1 and day 7. M, HD, EIP, OS and GIP are the names of the metabolic pathways in KEGG annotation. M, metabolism; HD, human diseases; EIP, environmental information processing; OS, organismal systems; GIP, genetic information processing. 

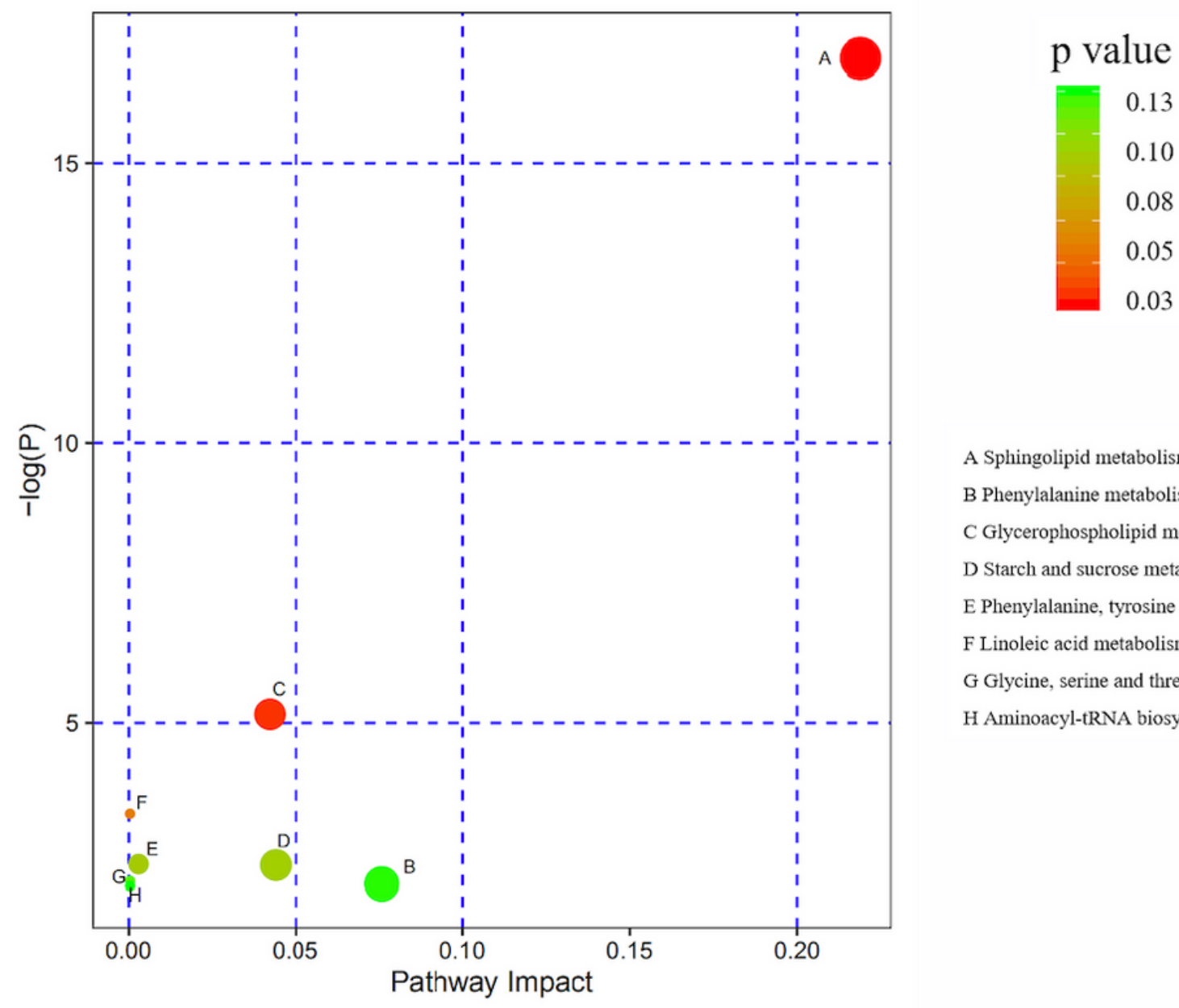

\begin{abstract}
A Sphingolipid metabolism
B Phenylalanine metabolism

C Glycerophospholipid metabolism

D Starch and sucrose metabolism

E Phenylalanine, tyrosine and tryptophan biosynthesis

F Linoleic acid metabolism

G Glycine, serine and threonine metabolism

H Aminoacyl-tRNA biosynthesis
\end{abstract}

\title{
Figure 6
}

Metabolome mapping of the differences in metabolite expression from day 1 to day 7 . The abscissa shows pathway impact and the ordinate gives the $\mathrm{p}$ value. The bigger the circles the greater the number of metabolites enriched in the pathway. Darker colors indicate smaller $p$ values.

\section{Supplementary Files}

This is a list of supplementary files associated with this preprint. Click to download.

- renamede61c7.docx 